\title{
Antifungal prophylaxis for prevention of COVID-19-associated pulmonary aspergillosis in critically ill patients: an observational study
}

Stefan Hatzl ${ }^{1,2+}$, Alexander C. Reisinger ${ }^{1 \dagger}$, Florian Posch ${ }^{3}$, Juergen Prattes ${ }^{4}$, Martin Stradner ${ }^{5}$, Stefan Pilz ${ }^{6}$, Philipp Eller ${ }^{7}$, Michael Schoerghuber ${ }^{7}$, Wolfgang Toller ${ }^{7}$, Gregor Gorkiewicz ${ }^{8}$, Philipp Metnitz ${ }^{8}$, Martin Rief ${ }^{8}$, Florian Prüller ${ }^{9}$, Alexander R. Rosenkranz ${ }^{10}$, Thomas Valentin ${ }^{5}$, Robert Krause ${ }^{4^{*}}$, Martin Hoenig ${ }^{4,11 \dagger}$ and Gernot Schilcher ${ }^{1+}$

\begin{abstract}
Background: Coronavirus disease 19 (COVID-19)-associated pulmonary aspergillosis (CAPA) emerged as important fungal complications in patients with COVID-19-associated severe acute respiratory failure (ARF). Whether mould active antifungal prophylaxis (MAFP) can prevent CAPA remains elusive so far.

Methods: In this observational study, we included all consecutive patients admitted to intensive care units with COVID-19-associated ARF between September 1, 2020, and May 1, 2021. We compared patients with versus without antifungal prophylaxis with respect to CAPA incidence (primary outcome) and mortality (secondary outcome). Propensity score adjustment was performed to account for any imbalances in baseline characteristics. CAPA cases were classified according to European Confederation of Medical Mycology (ECMM)/International Society of Human and Animal Mycoses (ISHAM) consensus criteria.

Results: We included 132 patients, of whom 75 (57\%) received antifungal prophylaxis (98\% posaconazole). Ten CAPA cases were diagnosed, after a median of 6 days following ICU admission. Of those, 9 CAPA cases were recorded in the non-prophylaxis group and one in the prophylaxis group, respectively. However, no difference in 30-day ICU mortality could be observed. Thirty-day CAPA incidence estimates were $1.4 \%(95 \% \mathrm{Cl} 0.2-9.7)$ in the MAFP group and $17.5 \%$ (95\% Cl 9.6-31.4) in the group without MAFP $(p=0.002)$. The respective subdistributional hazard ratio (sHR) for CAPA incidence comparing the MAFP versus no MAFP group was of 0.08 ( $95 \% \mathrm{Cl} 0.01-0.63 ; p=0.017$ ).
\end{abstract}

Conclusion: In ICU patients with COVID-19 ARF, antifungal prophylaxis was associated with significantly reduced CAPA incidence, but this did not translate into improved survival. Randomized controlled trials are warranted to evaluate the efficacy and safety of MAFP with respect to CAPA incidence and clinical outcomes.

Keywords: ICU, CAPA, COVID-19-associated aspergillosis, Posaconazole, Mould prophylaxis

*Correspondence: robert.krause@medunigraz.at

†Stefan Hatzl, Alexander C. Reisinger, Martin Hoenigl and Gernot Schilcher contributed equally to this paper.

${ }^{4}$ Division of Infectious Diseases, Department of Internal Medicine, Medical University of Graz, Graz, Austria

Full list of author information is available at the end of the article

\section{Introduction}

Severe acute respiratory syndrome coronavirus 2 (SARS-CoV-2) disease 2019 (COVID-19)-associated acute respiratory failure contributes to a highly permissive inflammatory environment. This in turn favours fungal pathogenesis due to the release of danger-associated molecular patterns and collateral effects of host original author(s) and the source, provide a link to the Creative Commons licence, and indicate if changes were made. The images or other third party material in this article are included in the article's Creative Commons licence, unless indicated otherwise in a credit line to the material. If material is not included in the article's Creative Commons licence and your intended use is not permitted by statutory regulation or exceeds the permitted use, you will need to obtain permission directly from the copyright holder. To view a copy of this licence, visit http://creativecommons.org/licenses/by/4.0/. The Creative Commons Public Domain Dedication waiver (http://creativeco mmons.org/publicdomain/zero/1.0/) applies to the data made available in this article, unless otherwise stated in a credit line to the data. 
recognition pathways required for the activation of antiviral immunity [1]. In this context, COVID-19-associated pulmonary aspergillosis (CAPA) has been emerging as an important fungal complication of COVID-19 [1], affecting an average of $3.1 \%$ (range $0.7-7.7 \%$ ) [2-5] of patients hospitalized with COVID-19, 8.9\% (range 2.5-39\%) of patients admitted to the intensive care unit (ICU) [3, $6-11]$, and an average of $20.1 \%$ (range $3.2-38 \%$ ) of those requiring invasive ventilation $[6,7,12-14]$.

Diagnosis of CAPA is challenging in patients with COVID-19-associated ARDS, as clinical picture and radiological findings of CAPA resemble those of severe COVID-19 [15, 16], and blood tests lack sensitivity due to the primarily airway invasive growth of Aspergillus in non-neutropenic patients [15-17]. Testing of bronchoalveolar lavage (BAL) with fungal culture, galactomannan (GM), Aspergillus polymerase chain reaction (PCR), or the Aspergillus GM lateral flow assay (LFA) is therefore preferred $[18,19]$, but due to the presumed risk of COVID-19 transmission through bronchoscopies, sampling of the primary infection site is still not performed consistently across ICUs.

The high prevalence rates of CAPA in critically ill patients requiring invasive ventilation together with the difficulties in diagnosis and the devastating overall mortality rates of over $50 \%[2-4,6,9-12,14,15,20,21]$ could justify clinical trials evaluating antifungal prophylaxis in COVID-19 patients with acute respiratory failure. One retrospective single-centre case series from Belgium has reported the successful use of prophylaxis in terms of CAPA case reduction with inhaled liposomal Amphotericin B in a cohort of ICU patients with severe COVID19 [6]; however, studies evaluating systemic antifungal prophylaxis are lacking.

The objective of this observational single-centre study was to evaluate the effectiveness of mould-active antifungal prophylaxis in preventing CAPA in critical care patients with COVID-19-associated acute respiratory failure. Secondary objectives included the evaluation of a potential survival benefit associated with antifungal prophylaxis as well as the impact of CAPA on overall survival.

\section{Methods}

\section{Study cohort}

We performed an observational study, enrolling all consecutive adult SARS-CoV-2 polymerase chain reaction (SARS-CoV-2 PCR) positive patients to our ICUs at the Department of Internal Medicine, Medical University of Graz, Austria (later referred to as ICU 1), or the Department of Anaesthesiology, Medical University of Graz, Austria (later referred to as ICU 2), between September 1, 2020, and May 1, 2021.
All patient data were uniformly collected as described previously [22, 23]. Therefore, laboratory, clinical and radiology data were extracted from our in-house electronic healthcare database system and inserted in a predefined electronic case report form (eCRF) using REDCap electronic data capture [24, 25]. For classification of CAPA, the 2020 European Confederation of Medical Mycology (ECMM)/International Society of Human and Animal Mycoses (ISHAM) consensus criteria were used [16]. According to these criteria, patients were categorized as either proven, probable or possible pulmonary and/or tracheobronchial CAPA, or no evidence for CAPA. In those receiving antifungal prophylaxis, CAPA cases were further classified according to the ECMM/ MSG definitions for breakthrough infections [26]. To reduce confirmation bias, CAPA classification was made by two infectious disease specialists who were blinded against the baseline characteristics and the administration of mould active antifungal prophylaxis.

Data analysis was performed after exclusion of fourteen patients who did not meet the predefined inclusion criteria or met a predefined exclusion criterion after initial screening (Fig. 1).

The research project was approved by the local institutional review board (EC \#32-296 ex 19/20).

\section{Antifungal prophylaxis group and control group}

Assignment to antifungal prophylaxis was informed by our in-house recommendation, which recommended antifungal prophylaxis with posaconazole or a similar mould active antifungal drug for every COVID-19 patient with acute respiratory failure admitted to the ICU. This recommendation was based on our experience with COVID-19-associated mould infections occurring before September 2020 [16, 27, 28] and on frequent fungal resistance testing within our centre which revealed no case of azol resistance in Aspergillus spp. to date. This recommendation was first implemented at ICU 1 and later at ICU 2. However, the decision of administration of mould active antifungal prophylaxis largely relays on the treating intensivist allowing us to compare outcomes of patients with and without antifungal prophylaxis who were otherwise treated according to same medical standards. Treated patients and control patients were distributed among both intensive care units.

\section{Statistical analysis}

All statistical analyses were performed using Stata (Windows version 16.1; Stata Corp., Houston, TX, USA) and R 4.0.5 (https://www.r-project.org/) and followed an a priori specified analysis plan (Additional file 1: Figure S1). The distribution of baseline variables between the antifungal prophylaxis and no antifungal prophylaxis group 


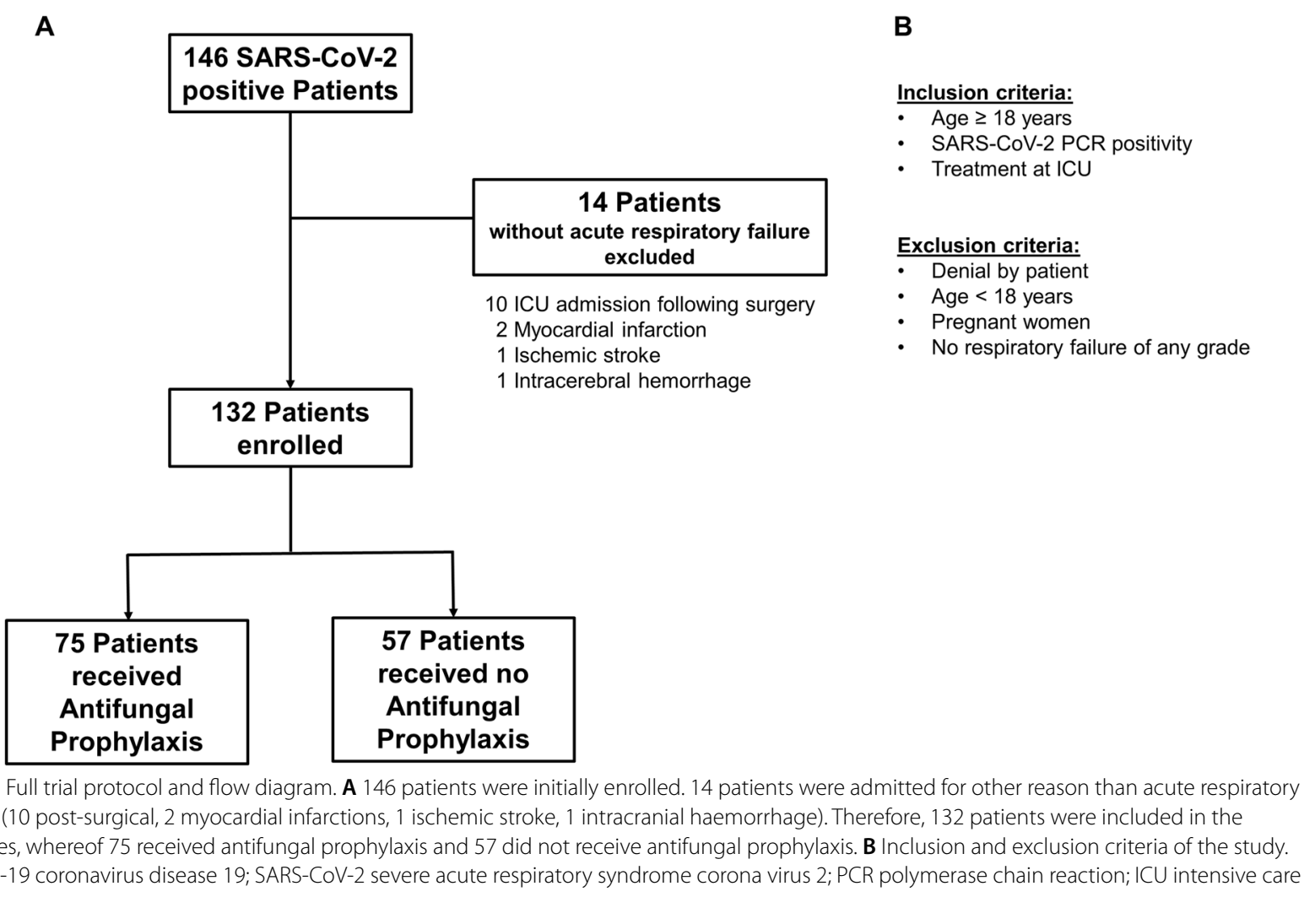

was evaluated using rank-sum tests, $\chi^{2}$-tests, and Fisher's exact tests, as appropriate. The magnitude of these differences was quantified with standardized mean differences (SMDs), and SMDs $\geq 0.31$ were considered indicative of a potentially relevant magnitude of difference. To account for differences in covariates between the groups with and without antifungal prophylaxis, we used propensity score analyses. We obtained the propensity score $e$ from a multivariable logistic regression model with antifungal prophylaxis group as the outcome variable. For this model, we pre-specified a fixed number of maximum 10 predictor variables, in order not to have less than 3 events per predictor variable. In detail, the model was developed by initially including all variables with a $\mathrm{p}$ for difference between the two groups of $\leq 0.16$ and/or a $S M D \geq 0.31$. The propensity score $e$ was then transformed into an inverse-probability-of-treatment-weight (IPTW) according to the average treatment effect principle, i.e. $=\frac{\text { (AntifungalProphylaxis) }}{e}+\frac{1-\text { (AntifungalProphylaxis) }}{1-e}$, where antifungal prophylaxis denotes the treatment assignment[29]. For balance diagnostics, we then re-estimated SMDs and p-values for difference between the two treatment groups with the IPTW-weighted data [30, 31]. For this propensity score model, missing data of ten variables (body mass index, $\mathrm{paO}_{2} / \mathrm{FiO}_{2}$, positive end expiratory pressure, lactate, interleukin 6 , C-reactive protein, ferritin, high sensitive troponin $\mathrm{T}, \mathrm{D}$-Dimer, absolute lymphocyte count) were imputed using a chained equations algorithm [32]. Between-group tests for differences were re-performed after weighting with the IPTW.

Sensitivity analyses included a trimmed IPTW (i.e. excluding patients with an IPTW $\leq$ the 1 st and $\geq$ the 99th percentile of its distribution).

The primary outcome of the study, occurrence of CAPA within 30-days of ICU admission, was defined as the time interval from ICU admission to the time point when the first positive sample for CAPA was obtained or the censoring date which was set the latest at 30 days from ICU admission. Survival time was inflated by one day in patients who died at the day of ICU admission $(n=2)$. The secondary outcome of the study, 30-day ICU overall survival (OS), was defined as the time interval from ICU admission to death-from-any-cause or the censoring date when being still alive 30 days after ICU admission.

In addition, we also analysed 90-day ICU overall survival to evaluate long-term ICU survival.

Univariable unweighted and IPTW-weighted Cox proportional hazards models and Fine and Gray proportional 
sub-distribution hazards models were fitted for analysing the association between antifungal prophylaxis and timeto-event end points, respectively.

Univariable unweighted and IPTW-weighted risks of development of CAPA and death-from-any-cause were analysed with competing risk cumulative incidence estimators, Gray's tests, and Fine and Gray proportional sub-distribution hazards models, respectively. Deathfrom-any-cause was considered as competing event of interest in the analyses of CAPA development risk [33].To eliminate immortal time bias in the analysis of the association of CAPA with ICU overall survival, the occurrence of CAPA was modelled as a time-dependent variable within Cox models. The association of CAPA with ICU survival was defined as co-secondary outcome. This was achieved by partitioning the follow-up time of patients who developed CAPA into times before and after CAPA diagnosis. For visual display of the association between CAPA and ICU overall survival outcomes, we performed a landmark analysis after 14 days of ICU admission. This landmark was chosen because most CAPA diagnosis happened in the first 2 weeks after ICU admission.

The full dataset and the main analysis code are available upon request by the first author.

In all analyses $p$ values $<0.05$ were considered statistically significant.

\section{Results}

\section{Cohort description and baseline characteristics}

Over the study period, 132 patients were admitted to the ICUs due to COVID-19-associated acute respiratory failure and therefore included in the final analysis (Fig. 1). Of those, 75 (57\%) patients received mould active antifungal prophylaxis within the first $48 \mathrm{~h}$ after ICU admission according to our local standard, while the remaining 57 (43\%) did not. At the time of ICU admission, the median age of patients was 65 years [25th-75th percentile: 55-75], and 48 (36\%) patients were female. The investigated population had a median of two coexisting conditions [1-4] and a median time from SARSCoV-2 PCR positivity to ICU admission of 3 days [0-6]. All patients included in this study showed an oxygen saturation of less than $88 \%$ while breathing ambient air prior to ICU admission and were classified as acute respiratory failure. Most patients exhibited severe acute respiratory failure as displayed by a median $\mathrm{paO} 2 / \mathrm{FiO} 2$ ratio (Horowitz Index) of 95 [25th-75th percentile 75-146]. Seventy-two patients (56\%) required invasive ventilation including veno-venous extracorporeal membrane oxygenation (vvECMO) $(n=13)$ and/or endotracheal intubation $(n=59)$. All patients in this study received systemic glucocorticoids as supportive treatment. Remdesivir was administered in $33(40 \%)$ patients, convalescent plasma was given to $50(38 \%)$ patients, whereas only one patient received tocilizumab (Table 1). During the first 30-days after ICU admission, we observed 10 CAPA cases, corresponding to 1-, 15-, and 30-day CAPA incidence of $0.8 \%$ (95\% CI 0.1-5.2), 8.5\% (4.6-15.2), and 8.5\% (4.6-15.2), respectively, in the complete cohort (Additional file 2: Figure S2).

\section{Mould active antifungal prophylaxis}

Out of 75 patients receiving antifungal prophylaxis, 73 (98\%) received standard dosage of intravenous posaconazole as mould active antifungal prophylaxis as recommended by our in-house protocol. One patient $(1 \%)$ received intravenous isavuconazole due to impaired tolerance against posaconazole. One patient $(1 \%)$ received caspofungin due to a suspected prior anaphylactic reaction against azoles. All patients within the prophylaxis group were started on antifungal prophylaxis within 48 -h after ICU admission (Table 1). At both ICU's routine serum-galactomannan and bronchoalveolar lavage-galactomannan testing was performed, and no differences in the frequencies of GM testing was observed between the prophylaxis and no prophylaxis group excluding any observation bias (Additional file 3: Table S1).

\section{Propensity score}

As outlined in Table 1, patients in the prophylaxis group had a lower body mass index, higher rates of chronic kidney disease, higher serum levels of creatinine and haemodialysis, lower rates of coronary artery disease, a higher proportion of immunosuppression, lower sequential organ failure score (SOFA), less frequencies in mask oxygen supply, lower levels of D-Dimer at ICU admission and received more frequently remdesivir as treatment for COVID-19 (Additional file 4: Figure S3). These imbalances between the treatment groups might display a non-random assignment bias. We pre-specified a propensity score model including a maximum of 10 variable; however, only 9 met the criteria of $p \leq 0.16$ and/ or $\mathrm{SMD} \geq 0.31$. Therefore, we predicted a propensity score based on a 9-variable multivariable logistic regression model (Additional file 5: Table S2) to control for the between group differences. The propensity score covered the whole probability range (Additional file 6: Figure S4A) and was then transformed into the IPTW (Additional file 6: Figure S4B). The propensity score was able to eliminate all difference between the treatment groups after re-weighting of the data (Table 1, Additional file 4: Figure S3) which allowed us to correct for a potential non-random assignment bias and provide more reliable results. Moreover, the trimmed IPTW showed the same potential in reducing the between group differences 
Table 1 Baseline characteristics of the study population—distribution at ICU admission

\begin{tabular}{|c|c|c|c|c|c|c|}
\hline Variable & $\mathrm{n}$ (\%miss) & Overall $(n=132)$ & $\begin{array}{l}\text { Antifungal } \\
\text { prophylaxis } \\
(n=75)\end{array}$ & $\begin{array}{l}\text { No antifungal } \\
\text { prophylaxis }(n=57)\end{array}$ & $p$ & $p_{\text {IPTW }}$ \\
\hline \multicolumn{7}{|l|}{ Demographic variables } \\
\hline Age (years) & $132(0 \%)$ & $65[55-75]$ & $67[55-74]$ & $63[56-75]$ & 0.879 & 0.375 \\
\hline Female Gender & $132(0 \%)$ & $48(36 \%)$ & $24(32 \%)$ & $24(42 \%)$ & 0.232 & 0.632 \\
\hline $\mathrm{BMI}\left(\mathrm{kg} / \mathrm{m}^{2}\right)$ & $127(4 \%)$ & $28.6[25.4-33.2]$ & $27.8[24.5-32.8]$ & $29.6[25.9-33.7]$ & 0.139 & 0.285 \\
\hline \multicolumn{7}{|l|}{ Coexisting conditions } \\
\hline Number of coexisting conditions & $132(0 \%)$ & $2[1-4]$ & $2[1-3]$ & $2[1-4]$ & 0.629 & 0.640 \\
\hline Hypertension & $132(0 \%)$ & $93(71 \%)$ & $49(65 \%)$ & $44(77 \%)$ & 0.186 & 0.562 \\
\hline Diabetes & $132(0 \%)$ & $31(23 \%)$ & $20(27 \%)$ & $11(19 \%)$ & 0.323 & 0.454 \\
\hline Atrial fibrillation & $132(0 \%)$ & $22(17 \%)$ & $10(13 \%)$ & $12(21 \%)$ & 0.238 & 0.400 \\
\hline Coronary artery disease $\mathrm{s}^{\$}$ & $132(0 \%)$ & $21(16 \%)$ & $9(12 \%)$ & $12(21 \%)$ & 0.159 & 0.686 \\
\hline Congestive heart failure & $132(0 \%)$ & $20(15 \%)$ & $9(12 \%)$ & $11(19 \%)$ & 0.247 & 0.763 \\
\hline Peripheral arterial disease & $132(0 \%)$ & $17(13 \%)$ & $7(9 \%)$ & $10(17 \%)$ & 0.163 & 0.171 \\
\hline Thromboembolic disease & $132(0 \%)$ & $19(14 \%)$ & $10(13 \%)$ & $9(16 \%)$ & 0.690 & 0.230 \\
\hline Chronic kidney disease & $132(0 \%)$ & $35(26 \%)$ & $15(20 \%)$ & $20(35 \%)$ & 0.052 & 0.892 \\
\hline Dialysis & $132(0 \%)$ & $11(8 \%)$ & $2(3 \%)$ & $9(16 \%)$ & 0.007 & 0.656 \\
\hline COPD & $132(0 \%)$ & $16(12 \%)$ & $13(17 \%)$ & $3(5 \%)$ & 0.035 & 0.867 \\
\hline Asthma & $132(0 \%)$ & $15(11 \%)$ & $9(12 \%)$ & $6(11 \%)$ & 0.792 & 0.702 \\
\hline Prior cancer in complete remission & $132(0 \%)$ & $11(8 \%)$ & $6(8 \%)$ & $5(8 \%)$ & 0.874 & 0.917 \\
\hline Active malignancy & $132(0 \%)$ & $9(7 \%)$ & $6(8 \%)$ & $3(5 \%)$ & 0.537 & 0.644 \\
\hline Dementia & $132(0 \%)$ & $4(3 \%)$ & $2(3 \%)$ & $2(4 \%)$ & 0.780 & 0.844 \\
\hline Prior transplantation & $132(0 \%)$ & $10(8 \%)$ & $7(9 \%)$ & $3(5 \%)$ & 0.381 & 0.821 \\
\hline Immunosuppression † & $132(0 \%)$ & $17(13 \%)$ & $13(17 \%)$ & $4(8 \%)$ & 0.008 & 0.921 \\
\hline \multicolumn{7}{|l|}{ ICU risk stratification } \\
\hline SOFA (points) & $132(0 \%)$ & $5[4-8]$ & $4[4-7]$ & $5[4-10]$ & 0.057 & 0.987 \\
\hline $\mathrm{paO}_{2} / \mathrm{FiO}_{2}$ & $128(3 \%)$ & $95[75-146]$ & 90 [75-130] & $107[75-152]$ & 0.302 & 0.749 \\
\hline $\operatorname{PEEP}(\mathrm{mmHg})$ - maximum & $111(16 \%)$ & $11[9-12]$ & $10[9-12]$ & $11[10-12]$ & 0.599 & 0.464 \\
\hline $\begin{array}{l}\text { Acute respiratory failure grade (inspired by ARDS } \\
\text { Berlin } 2015 \text {-classification) }\end{array}$ & $132(0 \%)$ & - & - & - & 0.660 & 0.636 \\
\hline -severe & & $76(58 \%)$ & $46(61 \%)$ & $30(54 \%)$ & - & \\
\hline -moderate & & $45(34 \%)$ & $24(32 \%)$ & $21(37 \%)$ & - & \\
\hline -mild & & $10(8 \%)$ & $5(7 \%)$ & $6(9 \%)$ & - & \\
\hline Ventilation & $132(0 \%)$ & - & - & - & 0.146 & 0.776 \\
\hline -Intubated & & $59(45 \%)$ & 35 (47\%) & $24(42 \%)$ & - & \\
\hline -vvECMO & & $13(10 \%)$ & $6(8 \%)$ & $7(12 \%)$ & - & \\
\hline$-\mathrm{NIV}$ & & $49(37 \%)$ & $31(41 \%)$ & $18(32 \%)$ & - & \\
\hline$-\mathrm{HFNC}$ & & $4(3 \%)$ & $2(3 \%)$ & $2(3 \%)$ & - & \\
\hline -Mask oxygen supply & & $7(5 \%)$ & $1(1 \%)$ & $6(11 \%)$ & - & \\
\hline Any invasive ventilation & & $72(56 \%)$ & $41(55 \%)$ & $31(54 \%)$ & 0.974 & 0.947 \\
\hline \multicolumn{7}{|l|}{ Antimycotic substances } \\
\hline Duration form diagnosis to initiation (days) & $75(0 \%)$ & - & $4[1-7]$ & - & - & \\
\hline Antimycotic drug used & $75(0 \%)$ & - & - & - & - & \\
\hline -Posaconazole & & - & $73(98 \%)$ & - & - & \\
\hline -Isavuconazole & & - & $1(1 \%)$ & - & - & \\
\hline -Caspofungin & & - & $1(1 \%)$ & - & - & \\
\hline \multicolumn{7}{|l|}{ Laboratory values } \\
\hline Lactate $(\mathrm{mmol} / \mathrm{l})$ & $127(4 \%)$ & $1.2[0.9-1.9]$ & $1.2[0.9-1.9]$ & $1.4[0.9-1.9]$ & 0.980 & 0.462 \\
\hline IL-6 (pg/ml) & $114(14 \%)$ & 83 [28-258] & 76 [28-185] & 89 [29-258] & 0.448 & 0.907 \\
\hline CRP (mg/l) & $130(2 \%)$ & 112 [70-177] & 113 [76-196] & 112[58-151] & 0.237 & 0.573 \\
\hline
\end{tabular}


Table 1 (continued)

\begin{tabular}{|c|c|c|c|c|c|c|}
\hline Variable & n (\%miss) & Overall $(n=132)$ & $\begin{array}{l}\text { Antifungal } \\
\text { prophylaxis } \\
(n=75)\end{array}$ & $\begin{array}{l}\text { No antifungal } \\
\text { prophylaxis }(n=57)\end{array}$ & $p$ & $p_{\text {IPTW }}$ \\
\hline Ferritin $(\mathrm{ng} / \mathrm{ml})$ & $113(13 \%)$ & 1334 [635-2196] & 1459 [755-2164] & $1128[493-2306]$ & 0.361 & 0.240 \\
\hline hs-TnT (pg/ml) & $119(10 \%)$ & $24[11-61]$ & $22[11-58]$ & 27 [11-92] & 0.488 & 0.889 \\
\hline D-Dimer (mg/l) & $121(8 \%)$ & $1.8[0.9-4.7]$ & $1.5[0.9-3.4]$ & $2.4[1.1-7.7]$ & 0.134 & 0.343 \\
\hline Creatine (mg/dl) & $132(0 \%)$ & $1.1[0.81-1.42]$ & $1.0[0.8-1.4]$ & $1.2[0.8-1.7]$ & 0.139 & 0.998 \\
\hline Bilirubin total (mg/dl) & $132(0 \%)$ & $0.5[0.4-0.7]$ & $0.5[0.4-0.7]$ & $0.5[0.4-0.7]$ & 0.774 & 0.784 \\
\hline SARS-CoV-2-Antibody status at ICU admission & $132(0 \%)$ & - & - & - & 0.612 & 0.617 \\
\hline -positive & & $35(27 \%)$ & $20(27 \%)$ & 15 (26\%) & - & - \\
\hline -negative & & $33(25 \%)$ & $21(27 \%)$ & $12(21 \%)$ & - & - \\
\hline -not available & & $64(48 \%)$ & $34(46 \%)$ & $30(52 \%)$ & - & - \\
\hline \multicolumn{7}{|l|}{ Blood counts } \\
\hline Leukocytes [G/l] & $132(0 \%)$ & $9.4[6.8-13.3]$ & $9.4[7.0-13.4]$ & $9.2[6.4-13.3]$ & 0.646 & 0.606 \\
\hline Neutrophils [G/l] & $132(0 \%)$ & $8.4[6.0-11.6]$ & $8.3[6.0-11.6]$ & $9.2[6.0-11.9]$ & 0.834 & 0.322 \\
\hline Lymphocytes [G/l] & $131(1 \%)$ & $0.7[0.5-0.9]$ & $0.8[0.5-1.0]$ & $0.6[0.5-0.9]$ & 0.299 & 0.340 \\
\hline Thrombocytes [G/l] & $132(0 \%)$ & 221 [158-309] & 240 [161-335] & 208 [152-293] & 0.164 & 0.834 \\
\hline \multicolumn{7}{|l|}{ Specific Medication } \\
\hline Glucocorticoids § & $132(0 \%)$ & $132(100 \%)$ & $75(100 \%)$ & $57(100 \%)$ & 1.000 & 1.000 \\
\hline Remdesivir & $132(0 \%)$ & $53(40 \%)$ & $36(48 \%)$ & $17(30 \%)$ & 0.035 & 0.555 \\
\hline Tocilizumab & $132(0 \%)$ & $1(1 \%)$ & $1(1 \%)$ & $0(0 \%)$ & 0.382 & 0.326 \\
\hline Convalescent Plasma & $132(0 \%)$ & $50(38 \%)$ & $32(43 \%)$ & $18(32 \%)$ & 0.193 & 0.871 \\
\hline \multicolumn{7}{|l|}{ Outcomes } \\
\hline CAPA & $132(0 \%)$ & & & & 0.008 & 0.015 \\
\hline -probable CAPA & - & $9(7 \%)$ & $1(1 \%)$ & $8(14 \%)$ & & \\
\hline -possible CAPA & - & $1(1 \%)$ & $0(0 \%)$ & $1(2 \%)$ & & \\
\hline Deceased at data cut off & $132(0 \%)$ & $65(49 \%)$ & $39(52 \%)$ & $26(46 \%)$ & 0.467 & - \\
\hline Length of ICU stay & $132(0 \%)$ & $13[5-25]$ & $13[6-24]$ & $12[4-25]$ & 0.700 & - \\
\hline
\end{tabular}

Data are reported as medians [25th-75th percentile] or as absolute counts (\%)

$p$ denotes $p$ values before ITPW weighting, $p_{\text {IPTW }}$ denotes $p$ values after IPTW adjustment

$p$ values are either from rank-sum tests, $\mathrm{X}^{2}$-tests, or Fisher's exact tests, as appropriate

The discrepancy between missing variable regarding $\mathrm{pO}_{2} / \mathrm{FiO}_{2}$ and $\mathrm{ARDS}$ classification inspired by the Berlin 2015 classification arises from missing exact $\mathrm{pO}_{2} / \mathrm{FiO} 2$ values; however, ARDS was classified

Abbreviations: BMI body mass index; ICU intensive care unit; COPD chronic obstructive pulmonary disease; SOFA sequential organ failure assessment; PEEP positive end expiratory pressure; vvECMO veno-venous extracorporeal membrane oxygenation, NIV non-invasive ventilation, HFNC high flow nasal cannula; IL-6 interleukin 6; CRP C-reactive protein; hs-TnT high sensitive troponin T, SARS-CoV2 severe acute respiratory syndrome corona virus 2; CAPA coronavirus disease-associated pulmonary aspergillosis

\$ documented coronary heart disease either by specific coronary imaging or coronary angiography

${ }^{\dagger}$ comprises immunosuppressive medication (low dose of glucocorticoids are excluded) as well as diseases with severe immunosuppression

$\S$ Glucocorticoids included low-dose dexamethasone or equivalent doses of other glucocorticoids

showing a high reliability of the computed propensity score (data not shown).

\section{CAPA incidence and mould active antifungal prophylaxis}

During the first 30-days after ICU admission, we observed 10 CAPA cases ( 9 probable and 1 possible CAPA cases), corresponding to 1-, 15-, and 30-day CAPA incidence of $0.8 \%$ (95\% CI $0.1-5.2), 8.5 \%$ (4.6-15.2), and 8.5\% (4.6-15.2), respectively. No case of CAPA was diagnosed after 30-days of ICU admission. CAPA cases were diagnosed after a median
6 days following ICU admission (25th-75th percentile 3-9) and presented with positive BAL galactomannan $(\mathrm{GM})>1.0$ optical density index (ODI, 7/10), BAL culture growing Aspergillus species (4/10), positive serum GM $>0.5$ ODI $(4 / 10)$, and positive BAL Aspergillus PCR (5/10) in addition to the other required parameters defining CAPA. All patients analysed had at least on negative diagnostic test for CAPA according to the ECMM/ ISHAM consensus after ICU admission to exclude potential pre-emptive treatment. Patients diagnosed with CAPA were treated according 
the ECMM/ISHAM consensus. In detail, in the nonprophylaxis group 3 patients received isavuconazole and 6 received posaconazole, the patient in the prophylaxis group developing a breakthrough infection was switched to isavuconazole. Detailed characteristics of the CAPA patients are summarized in Additional file 7: Table S3.

Of the 10 CAPA cases, 9 occurred in patients not receiving anti-mould prophylaxis, while one occurred in the anti-mould prophylaxis group (one time serum GM positivity as only mycological criterion in that patient, while BAL GM was negative). With the unadjusted competing risk analysis, the 30-day CAPA incidence estimates were $17.5 \%$ (95\% CI 9.6-31.4) in the non-antifungal prophylaxis group and $1.4 \%$ (95\% CI $0.2-9.7)$ in those receiving anti-mould prophylaxis (Gray's test $p=0.002$, Fig. 2A). In the Fine and Gray's model, this corresponded to a sub-distributional hazard Ratio (SHR) of 0.08 (95\% CI $0.01-0.63, p=0.017$ ) in the group with versus without administration of mould active antifungal prophylaxis.

In addition, we performed an analysis using the reweighted data with the generated IPTW. After adjustment, the 30-day CAPA incidence estimates were $15.8 \%$ in the non-antifungal prophylaxis group and $1.1 \%$ in the mould active antifungal prophylaxis group (Gray's test $p=0.001$, Fig. 2B). In the Fine and Gray's model, this corresponded to a SHR of 0.07 (95\% CI 0.01-0.57, $p=0.013$ ) for administration of mould active antifungal prophylaxis.

\section{Univariable and multivariable predictors of CAPA}

Further univariable analyses of 30-day-CAPA identified higher positive end-expiratory pressure (HR per 1 $\mathrm{cmH}_{2} \mathrm{O}$ increase $=1.04,95 \%$ CI 1.02-1.07, $p=0.001$ ), endotracheal intubation (HR 4.89, 95\% CI 1.03-23.03, $p=0.045)$, and decreased absolute lymphocyte counts (HR per $1 \mathrm{G} / \mathrm{l}$ increase $=0.30,95 \%$ CI $0.10-0.93$, $p=0.037$ ) as factors associated with CAPA (Additional file 8: Table S4). To determine the independent prognostic value of mould active antifungal prophylaxis for 30-day-CAPA development, multivariable analyses including all univariable predictors were performed. Here, the prognostic association prevailed. (Additional file 9: Table S5).

\section{Mould active antifungal prophylaxis, CAPA, and ICU survival}

Within 30 days of ICU admission, 48 deaths were observed, corresponding to 1-, 15-, and 30-day ICU survival estimates of 96\% (95\% CI 91-98), 78\% (70-84), and $63 \%$ (54-71), respectively, in the whole cohort (Additional file 2: Fig. S2).

In the time to event analysis, patients receiving antifungal prophylaxis experienced no improvement of 30-day ICU survival outcomes: 1-, 15- and 30-day ICU survival rates were 98.6\% (95\% CI 90.1-99.8), 78.3\% (67.1-86.2) and $62.7 \%$ (50.5-72.6) in patients given mould active antifungal prophylaxis, and $93.0 \%$ (82.3-97.3), $77.1 \%$ (64.0-86.1) and $63.1 \%(49.2-74.1)$ in those not receiving mould active prophylaxis, respectively. The KaplanMeier analysis results revealed no differences between

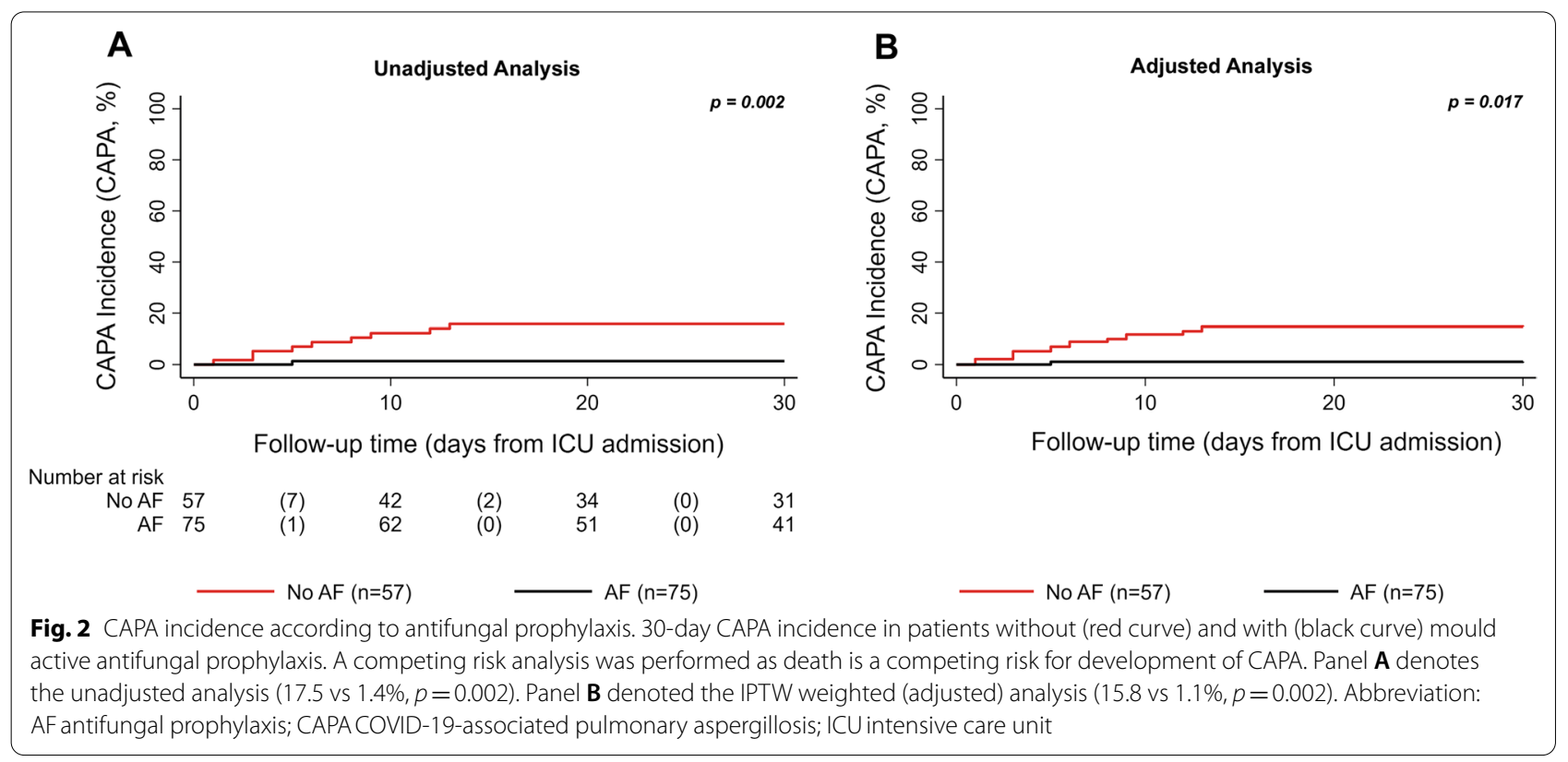


the treatment groups (log-rank $p=0.955)$ (Fig. 3A). To account for the non-random assignment bias, we also re-weighted this data for the IPTW. However, the reweighted analysis also showed no differences in 30-day ICU survival between patients receiving antifungal prophylaxis and those who did not $(\log$-rank $p=0.905)$ (Fig. 3B). To investigate a potential effect of mould-active antifungal prophylaxis on long-term ICU survival, we repeated the time-to-event analysis for 90-day ICU survival. Interestingly, no difference between both groups could be observed regarding this co-secondary endpoint (Additional file 10: Fig. S5).

Using a univariable time-to-event regression analysis treating development of CAPA as dependent variable, we also evaluated impact of CAPA on 90-day ICU survival as co-secondary outcome for long-term survival. Patients who developed CAPA during their ICU stay displayed worse outcomes regarding 90-day ICU mortality (HR 2.30, 95\% CI 1.08-4.91, $p=0.031$ ). These findings prevailed in multivariable regression analyses adjusting for prognostic co-variates, such as age, SOFA, creatinine, number of comorbidities, body-mass-index, immunosuppression, and supportive treatment with convalescent plasma as previously described (Additional file 11: Table S6). In a landmark analysis after 14 days, 90-day ICU survival estimates were $63.8 \%$ and $16.7 \%$ in patients in patients with and without CAPA during their ICU stay (Mantel-Byar $p=0.026$, Fig. 4).

\section{Discussion}

We observed that mould-active antifungal prophylaxis for patients with COVID-19-associated acute respiratory failure admitted to the ICU was highly effective

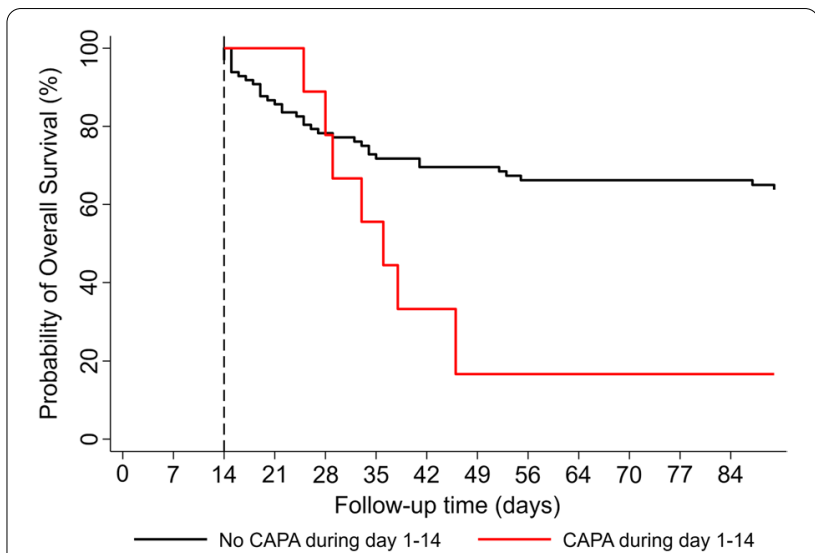

Fig. 4 Landmark analysis of 90-day ICU survival according to CAPA diagnosis. Day 14 after ICU admission was chosen as landmark date, as most CAPA diagnosis occurred before this time point. 90-day overall survival estimates were $52.9 \%$ in patients without CAPA and $14.6 \%$ in patients with CAPA $(p=0.026)$. Abbreviation: CAPA COVID-19-associated pulmonary aspergillosis; ICU intensive care unit

in preventing CAPA. In patients without antifungal prophylaxis, CAPA was diagnosed in $17.5 \%$ versus only in $1.4 \%$ of those receiving prophylaxis, representing a reduction in relative risk for development of CAPA of $92 \%$ in the prophylaxis group and a number needed to receive prophylaxis of 7 to prevent one CAPA case. These results outline the efficacy of prophylaxis with posaconazole. While CAPA was associated with significantly higher mortality, antifungal prophylaxis did not have a significant impact on overall survival.

In our study, mould active antifungal prophylaxis resulted in a $>90 \%$ reduction of CAPA, outlining the

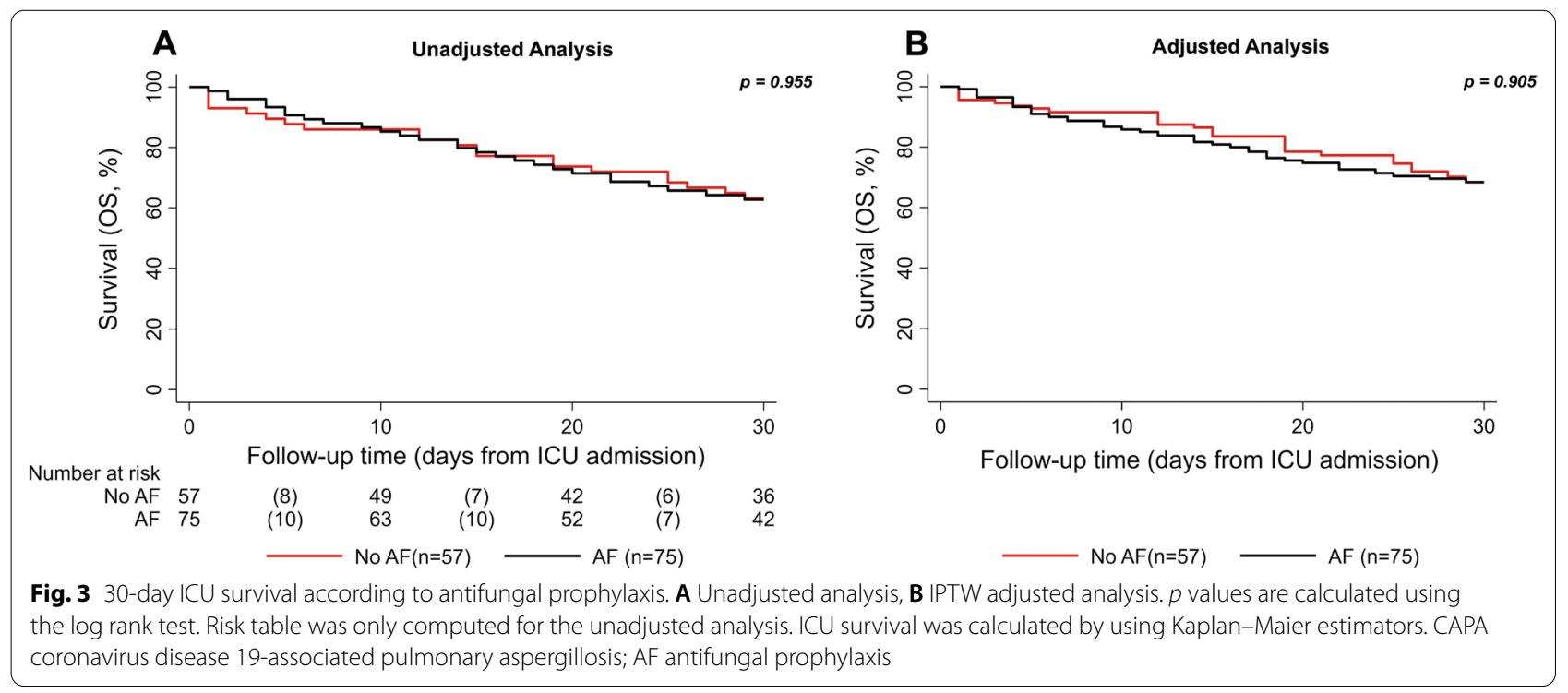


effectiveness of antimould prophylaxis in this setting. After adjusting for differences in baseline characteristics, the positive effect of mould active antifungal prophylaxis was even stronger. The vast majority of patients in our study received posaconazole prophylaxis, the current gold standard for antifungal prophylaxis in patients with haematological malignancies [34, 35]. Posaconazole and particularly its newer tablet and intravenous formulations have several advantages compared to other drugs that are used for prophylaxis, starting with favourable pharmacokinetics, long half live and high intracellular concentrations [36]. Voriconazole and isavuconazole are predominantly metabolized by the CYP enzyme system resulting in higher rates of drug-drug interactions, when compared to posaconazole which is metabolized predominantly through the uridine diphosphate-glucuronyltransferase enzyme pathways [37-39]. Voriconazole is in fact among the drugs most frequently associated with major drug-drug interactions in the ICU setting [40], and may also show interactions with remdesivir which also is a substrate for CYP3A4, although its metabolism is primarily mediated by hydrolase activity [41]. Administration of intravenous liposomal Amphotericin B is complicated by the fact that SARS-CoV-2 has shown renal tropism and has been described as a frequent cause of acute kidney injury [42]. Inhaled amphotericin B may be an alternative for prophylaxis of CAPA but lacks regulatory approval in this application and has been associated with severe adverse events [43]. Therefore, posaconazole seems for now an appropriate choice for CAPA prophylaxis, although isavuconazole may be an alternative. New antifungal classes currently under development, namely fosmanogepix and olorofim, which show equal efficacy with minimal drug-drug interactions and toxicity in current phase IIb studies may become options/alternatives soon.

Using antimould prophylaxis for prevention of CAPA is attractive, because CAPA has been associated with high mortality rates $[2-4,12,15]$, which was confirmed in our study where patients who developed CAPA during their ICU stay displayed worse outcomes with a more than twofold higher mortality compared to patients without CAPA. Given that patients who succumb very early after ICU admission are no longer able to develop CAPAand most diagnoses occurred within 14 days after ICU admission in our cohort (median time to diagnosis 6 days after ICU admission) - we performed a landmark analysis from this time point and found that survival estimates were $64 \%$ in patients without CAPA compared to $17 \%$ in those diagnosed with CAPA. While early treatment may reduce mortality [2], early diagnosis of CAPA is challenging because clinical presentation and imaging findings of COVID-19 and CAPA may overlap (fever, shortness of breath, cough; unspecific infiltrates and consolidations, halo sign), and serum galactomannan has a low sensitivity $[1,15]$. While prolonged neutropenia, stem-cell transplantation, immunosuppression, underlying lung disease, or systemic corticosteroids have been described as risk factors for development of CAPA [1], none of those is a strong predictor of CAPA, which may very well also develop in patients without any other risk factors than COVID-19-associated acute respiratory failure [1].

Pulmonary aspergillosis may also complicate acute respiratory failure caused by other viral infections, among those with severe influenza where we have recently observed three cases of pulmonary aspergillosis in our centre [19].

In a recent study on anti-mould prophylaxis to prevent influenza-associated aspergillosis [44], posaconazole did not show significant benefit due to the fact that patients developed invasive aspergillosis mostly within $48 \mathrm{~h}$ of ICU admission (i.e. before posaconazole reaches its steady state [45]). In contrast, CAPA has been shown before to develop mostly $>5$ days after ICU admission, by which time steadystate posaconazole levels are usually reached [15, 46, 47], making it a better target for antifungal prophylaxis.

Importantly, the prevalence of CAPA has been shown to vary between centres. The prevalence of $17.5 \%$ observed in our cohort not receiving anti-mould prophylaxis was higher than the median $8.9 \%$ reported previously for COVID-19 patients admitted to ICU [3, 6-11, 20, 48, 49], but has been lower than the median $20.1 \%$ reported in other studied for COVID-19 patients requiring invasive ventilation $[4,6,7,12-14]$. Whether or not the $>90 \%$ efficacy in reducing CAPA shown in our study will justify the broad use of antimould prophylaxis in other centres will mainly depend on local prevalence rates of CAPA. Targeted use of prophylaxis in certain groups of patients at higher risk may also be an option for some centres.

Our study has several limitations including those patients were not randomized to receive mould active antifungal prophylaxis, and cases and controls were enrolled-in part-at different ICUs. However, we found similar baseline characteristics in patient with or without mould active antifungal prophylaxis, and additionally adjusted for any imbalances using a propensity score and IPTW weighted analysis. Also, this is a single-centre study and although the observed prevalence in our centre was within the range reported before, the reported range is wide and prevalence of CAPA observed in our centre may not translate to other centres.

\section{Conclusion}

In conclusion, this is the first study evaluating efficacy of mould active antifungal prophylaxis in ICU patients suffering from acute respiratory failure due to COVID19. We were able to show in our cohort that antifungal 
prophylaxis was able to prevent CAPA. The occurrence of CAPA was found to be associated with poor outcomes.

\section{Supplementary Information}

The online version contains supplementary material available at https://doi. org/10.1186/s13054-021-03753-9.

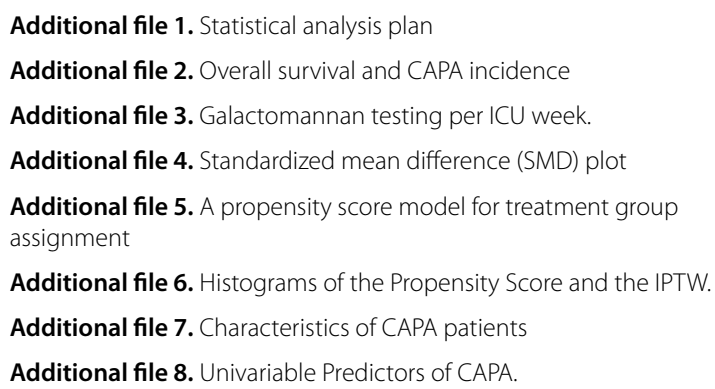

Additional file 9. Multivariable Cox regression of overall survival according to antifungal prophylaxis in the overall cohort

Additional file 10. 90-day ICU survival according to antifungal prophylaxis

Additional file 11. A multivariable cox regression model for 90-day ICU survival for adjustment of post-event CAPA for 7 important predictors of ICU survival

\section{Author's contributions}

SH, RK and GS planned and designed the study. SH, ACR, MR, TV, RK and GS collected the data. SH and FP analysed the data. SH and FP developed the methodology and the analysis plan. SH administered the project. $\mathrm{RK}, \mathrm{MH}$ and GS supervised the project. SH, ACR, MH, TV, RK and GS validated the data. SH visualized the data. SH, ACR and MH wrote the original draft. SH, ACR, FP, JP, MS, SP, PE, MS, WT, GG, PM, MR, FPr, ARR, TV, RK, MH and GS critically revised the manuscript. SH, ACR and FP verified the underlying data. All authors read and approved the final manuscript.

\section{Funding}

The study was supported by a research grant of the City of Graz to S. Hatzl. M. Hoenigls efforts were supported by the NIH (Project \# UL1TR001442-061) as well as investigator-initiated research grants from Astellas, MSD and Pfizer. J. Prattes efforts were supported by a grant from MSD. The funders had no impact on the study design, data collection and analysis, decision to publish, or preparation of the manuscript.

\section{Availability of data and materials}

The blinded analysed dataset is available on request by the first author. Email: stefan.hatzl@medunigraz.at.

\section{Code availability}

The full analysis code (STATA) is available on request by the first author. Email: stefan.hatzl@medunigraz.at.

\section{Declarations}

\section{Ethical approval}

All procedures performed in studies involving human participants were in accordance with the ethical standards of the institutional and/or national research committee and with the 1964 Helsinki declaration and its later amendments or comparable ethical standards. Approval of the local institutional review board: (EC \#32-296 ex 19/20) was obtained.

\section{Consent to participate}

Not applicable.

\section{Consent for publication}

Not applicable.

\section{Conflict of interest}

S. Hatzl declares no conflict of interest. A.C. Reisinger declares no conflict of interest. F. Posch declares no conflict of interest. J. Prattes received grants from Pfizer, MSD, Gilead; honoraria from Menarini, MSD, Angelini, Pfizer, Gilead; participated in advisory boards from Pfizer and holds stocks and stock-options from AbbVie Inc., NovoNordisk, Stryker. M. Stradner declares no conflict of interest. S. Pilz declares no conflict of interest. P. Eller declares no conflict of interest. M. Schoerghuber declares no conflict of interest. W. Toller declares no conflict of interest. G. Gorkiewicz declares no conflict of interest. P. Metnitz declares no conflict of interest. M. Rief declares no conflict of interest. F. Prüller declares no conflict of interest. A.R. Rosenkranz declares no conflict of interest. T. Valentin declares no conflict of interest. R. Krause declares no conflict of interest. M. Hoenigl received grants from Astellas, MSD, Pfizer, Gilead. G. Schilcher declares no conflict of interest. S. Hatzl and M. Hoenigl received independent (non-industrial affiliated) grant from the National Institute of Health (M. Hoenigl) and the City of Graz (S. Hatzl).

\section{Author details}

${ }^{1}$ Intensive Care Unit, Department of Internal Medicine, Medical University of Graz, Graz, Austria. ${ }^{2}$ Division of Haematology, Department of Internal Medicine, Medical University of Graz, Graz, Austria. ${ }^{3}$ Division of Oncology, Department of Internal Medicine, Medical University of Graz, Graz, Austria. ${ }^{4}$ Division of Infectious Diseases, Department of Internal Medicine, Medical University of Graz, Graz, Austria. ${ }^{5}$ Division of Rheumatology and Immunology, Department of Internal Medicine, Medical University of Graz, Graz, Austria. ${ }^{6}$ Division of Endocrinology and Diabetology, Department of Internal Medicine, Medical University of Graz, Graz, Austria. ${ }^{7}$ Department of Anaesthesiology and Intensive Care Medicine, Medical University Graz, Graz, Austria. ${ }^{8}$ Institute of Pathology, Medical University of Graz, Graz, Austria. ${ }^{9}$ Clinical Institute for Medical and Chemical Laboratory Diagnostics, Medical University of Graz, Graz, Austria. ${ }^{10}$ Division of Nephrology, Department of Internal Medicine, Medical University of Graz, Graz, Austria. ${ }^{11}$ Division of Infectious Diseases, University of California San Diego, San Diego, USA.

Received: 28 June 2021 Accepted: 31 August 2021

Published online: 15 September 2021

\section{References}

1. Arastehfar A, Carvalho A, van de Veerdonk FL, Jenks JD, Koehler P, Krause R, et al. COVID-19 Associated Pulmonary Aspergillosis (CAPA)-from immunology to treatment. J Fungi (Basel). 2020;6(2):91.

2. Bartoletti M, Pascale R, Cricca M, Rinaldi M, Maccaro A, Bussini L, et al. Epidemiology of invasive pulmonary aspergillosis among COVID-19 intubated patients: a prospective study. Clin Infect Dis. 2020.

3. Nasir N, Farooqi J, Mahmood SF, Jabeen K. COVID-19-associated pulmonary aspergillosis (CAPA) in patients admitted with severe COVID-19 pneumonia: an observational study from Pakistan. Mycoses. 2020;63(8):766-70.

4. van Arkel ALE, Rijpstra TA, Belderbos HNA, van Wijngaarden P, Verweij PE, Bentvelsen RG. COVID-19 Associated Pulmonary Aspergillosis. Am J Respir Crit Care Med. 2020;202:132.

5. Segrelles-Calvo G, Araújo GRS, Llopis-Pastor E, Carrillo J, HernándezHernández M, Rey L, et al. Prevalence of opportunistic invasive aspergillosis in COVID-19 patients with severe pneumonia. Mycoses. 2021;64(2):144-51.

6. Rutsaert L, Steinfort N, Van Hunsel T, Bomans P, Naesens R, Mertes H, et al. COVID-19-associated invasive pulmonary aspergillosis. Ann Intensive Care. 2020;10(1):71.

7. Lamoth F, Glampedakis E, Boillat-Blanco N, Oddo M, Pagani JL. Incidence of invasive pulmonary aspergillosis among critically ill COVID-19 patients. Clin Microbiol Infect. 2020;26:1706.

8. Roman-Montes CM, Martinez-Gamboa A, Diaz-Lomelí P, Cervantes-Sanchez A, Rangel-Cordero A, Sifuentes-Osornio J, et al. Accuracy of galactomannan testing on tracheal aspirates in COVID-19-associated pulmonary aspergillosis. Mycoses. 2021;64(4):364-71. 
9. White PL, Dhillon R, Cordey A, Hughes H, Faggian F, Soni S, et al. A national strategy to diagnose COVID-19 associated invasive fungal disease in the ICU. Clin Infect Diseases. 2020.

10 Koehler PCO, Böttiger BW, Dusse F, Eichenauer DA, Fuchs F, Hallek M. COVID19 Associated Pulmonary Aspergillosis. Mycoses. 2020;63:528.

11. Machado M, Valerio M, Álvarez-Uría A, Olmedo M, Veintimilla C, Padilla B, et al. Invasive pulmonary aspergillosis in the COVID-19 era: An expected new entity. Mycoses. 2021;64(2):132-43.

12. Alanio A, Dellière S, Fodil S, Bretagne $S$, Mégarbane B. Prevalence of putative invasive pulmonary aspergillosis in critically ill patients with COVID-19. Lancet Respir Med. 2020;8(6)::48-9.

13. Gangneux JP, Reizine F, Guegan H, Pinceaux K, Le Balch P, Prat E, et al. Is the COVID-19 pandemic a good time to include aspergillus molecular detection to categorize aspergillosis in ICU patients? A monocentric experience. J Fungi (Basel). 2020;6(3):105.

14. Dupont D, Menotti J, Turc J, Miossec C, Wallet F, Richard J-C, et al. Pulmonary aspergillosis in critically ill patients with Coronavirus Disease 2019 (COVID19). Med Mycol. 2021;59(1):110-4.

15. Salmanton-García J, Sprute R, Stemler J, Bartoletti M, Dupont D, Valerio M, et al. COVID-19-Associated Pulmonary Aspergillosis, March-August 2020. Emerg Infect Dis. 2021;27(4):1077-86.

16. Koehler P, Bassetti M, Chakrabarti A, Chen SCA, Colombo AL, Hoenigl M, et al. Defining and managing COVID-19-associated pulmonary aspergillosis: the 2020 ECMM/ISHAM consensus criteria for research and clinical guidance. Lancet Infect Diseases. 2020;21:e149.

17. Arastehfar A, Carvalho A, Houbraken J, Lombardi L, Garcia-Rubio R, Jenks $J D$, et al. Aspergillus fumigatus and aspergillosis: from basics to clinics. Stud Mycol. 2021;100:100115

18. Jenks JD, Nam HH, Hoenigl M. Invasive aspergillosis in critically ill patients: Review of definitions and diagnostic approaches. Mycoses. 2021;64:1002.

19. Jenks JD, Prattes J, Frank J, Spiess B, Mehta SR, Boch T, et al. Performance of the bronchoalveolar lavage fluid aspergillus galactomannan lateral flow assay with cube reader for diagnosis of invasive pulmonary aspergillosis: a Multicenter Cohort Study. Clinical Infectious Diseases. 2020

20. Chauvet P, Mallat J, Arumadura C, Vangrunderbeek N, Dupre C, Pauquet $P$, et al. Risk factors for invasive pulmonary aspergillosis in critically ill patients with coronavirus disease 2019-induced acute respiratory distress syndrome. Crit Care Explor. 2020;2(11):e0244.

21. Marr K, Platt A, Tornheim J, Zhang S, Datta K, Cardozo C, et al. Aspergillosis complicating severe coronavirus disease. Emerg Infect Disease J. 2021;27(1):18.

22. Hatzl S, Posch F, Sareban N, Stradner M, Rosskopf K, Reisinger AC, et al. Convalescent plasma therapy and mortality in COVID-19 patients admitted to the ICU: a prospective observational study. Ann Intensive Care. 2021;11(1):73.

23. Hatzl S, Eisner F, Schilcher G, Kreuzer P, Gornicec M, Eller P, et al. Response to "COVID-19 in persons with haematological cancers." Leukemia. 2020;34(8):2265-70

24. Harris PA, Taylor R, Minor BL, Elliott V, Fernandez M, O'Neal L, et al. The REDCap consortium: building an international community of software platform partners. J Biomed Inform. 2019:95:103208.

25. Harris PA, Taylor R, Thielke R, Payne J, Gonzalez N, Conde JG. Research electronic data capture (REDCap)—A metadata-driven methodology and workflow process for providing translational research informatics support. J Biomed Inform. 2009:42(2):377-81.

26. Cornely OA, Hoenigl M, Lass-Flörl C, Chen SC, Kontoyiannis DP, Morrissey $\mathrm{CO}$, et al. Defining breakthrough invasive fungal infection-Position paper of the mycoses study group education and research consortium and the European Confederation of Medical Mycology. Mycoses. 2019;62(9):716-29.

27. Zurl C, Hoenigl M, Schulz E, Hatzl S, Gorkiewicz G, Krause R, et al. Autopsy proven pulmonary mucormycosis due to Rhizopus microsporus in a critically III COVID-19 patient with underlying hematological malignancy. J Fungi (Basel). 2021;7(2):88.

28 Prattes J, Valentin T, Hoenigl M, Talakic E, Reisinger AC, Eller P. Invasive pulmonary aspergillosis complicating COVID-19 in the ICU - A case report. Med Mycol Case Rep. 2020;31:2.

29. Austin PC. An introduction to propensity score methods for reducing the effects of confounding in observational studies. Multiv Behav Res, 2011;46(3):399-424.
30. Austin PCTJV. Bootstrap methods for developing predictive models. Am Stat. 2004;58(2):131-7.

31. White IR, Daniel R, Royston P. Avoiding bias due to perfect prediction in multiple imputation of incomplete categorical variables. Comput Stat Data Anal. 2010;54(10):2267-75.

32. White IR, Royston P, Wood AM. Multiple imputation using chained equations: Issues and quidance for practice. Stat Med. 2011;30(4):377-99.

33. Fine JP, Gray RJ. A proportional hazards model for the subdistribution of a competing risk. J Am Stat Assoc. 1999:94(446):496-509.

34. Cornely OA, Maertens J, Winston DJ, Perfect J, Ullmann AJ, Walsh TJ, et al. Posaconazole vs. fluconazole or itraconazole prophylaxis in patients with neutropenia. New Engl J Med. 2007;356(4):348-59.

35. Ullmann AJ, Lipton JH, Vesole DH, Chandrasekar P, Langston A, Tarantolo SR, et al. Posaconazole or fluconazole for prophylaxis in severe graft-versus-host disease. N Engl J Med. 2007;356(4):335-47.

36. Jenks JD, Mehta SR, Hoenigl M. Broad spectrum triazoles for invasive mould infections in adults: Which drug and when? Med Mycol. 2019;57(Supplement _2):S168-78.

37. Hope WW. Population pharmacokinetics of voriconazole in adults. Antimicrob Agents Chemother. 2012;56(1):526-31.

38. Brüggemann RJ, Alffenaar JW, Blijlevens NM, Billaud EM, Kosterink JG, Verweij PE, et al. Clinical relevance of the pharmacokinetic interactions of azole antifungal drugs with other coadministered agents. Clin Infect Diseases. 2009:48(10):1441-58.

39. Schmitt-Hoffmann A, Desai A, Kowalski D, Pearlman H, Yamazaki T, Townsend R. Isavuconazole absorption following oral administration in healthy subjects is comparable to intravenous dosing, and is not affected by food, or drugs that alter stomach $\mathrm{pH}$. Int $\mathrm{J}$ Clin Pharmacol Ther 2016;54(8):572-80.

40 Baniasadi S, Farzanegan B, Alehashem M. Important drug classes associated with potential drug-drug interactions in critically ill patients: highlights for cardiothoracic intensivists. Ann Intensive Care. 2015;5(1):44.

41. McCreary EK, Pogue JM, on behalf of the Society of Infectious Diseases P. Coronavirus Disease 2019 Treatment: A Review of Early and Emerging Options. Open Forum Infectious Diseases. 2020;7(4).

42. Puelles VG, Lütgehetmann M, Lindenmeyer MT, Sperhake JP, Wong MN, Allweiss L, et al. Multiorgan and Renal Tropism of SARS-CoV-2. New England Journal of Medicine. 2020

43. Bhaskaran A, Mumtaz K, Husain S. Anti-aspergillus prophylaxis in lung transplantation: a systematic review and meta-analysis. Curr Infect Disease Rep. 2013;15(6):514-25.

44. Vanderbeke L, Janssen NAF, Bergmans D, Bourgeois M, Buil JB, Debaveye $Y$, et al. Posaconazole for prevention of invasive pulmonary aspergillosis in critically ill influenza patients (POSA-FLU): a randomised, open-label, proofof-concept trial. Intensive Care Med. 2021:1-13.

45. Cornely OA, Hoenigl M, Lass-Florl C, Chen SC, Kontoyiannis DP, Morrissey $\mathrm{CO}$, et al. Defining breakthrough invasive fungal infection-Position paper of the mycoses study group education and research consortium and the European Confederation of Medical Mycology. Mycoses. 2019;62(9):716-29.

46. Reizine F, Pinceaux K, Lederlin M, Autier B, Guegan H, Gacouin A, et al. Influenza- and COVID-19-associated pulmonary aspergillosis: are the pictures different? J Fungi (Basel). 2021;7(5):388.

47. Prattes J, Duettmann W, Hoenigl M. Posaconazole plasma concentrations on days three to five predict steady-state levels. Antimicrob Agents Chemother. 2016;60(9):5595-9.

48. Zhang G, Hu C, Luo L, Fang F, Chen Y, Li J, et al. Clinical features and shortterm outcomes of 221 patients with COVID-19 in Wuhan. China J Clin Virol. 2020;127:104364

49. Chen X, Zhao B, Qu Y, Chen Y, Xiong J, Feng Y, et al. Detectable Serum Severe Acute Respiratory Syndrome Coronavirus 2 Viral Load (RNAemia) Is Closely Correlated With Drastically Elevated Interleukin 6 Level in Critically III Patients With Coronavirus Disease 2019. Clin Infect Diseases. 2020;71(8):1937-42.

\section{Publisher's Note}

Springer Nature remains neutral with regard to jurisdictional claims in published maps and institutional affiliations. 